\title{
Publisher's Note: Charge avalanches and depinning in the Coulomb glass: The role of long-range interactions [Phys. Rev. B 93, 094429 (2016)]
}

\author{
Juan Carlos Andresen, Yohanes Pramudya, Helmut G. Katzgraber, Creighton K. Thomas, \\ Gergely T. Zimanyi, and V. Dobrosavljević \\ (Received 10 June 2016; published 17 June 2016)
}

DOI: 10.1103/PhysRevB.93.219903

This paper was published online on 24 March 2016 with an error in the Acknowledgments on page 7 . The second sentence of the Acknowledgments should read as "V.D. and Y.P. were supported by the NSF (Grants No. DMR-1005751 and DMR-1410132)." The paper has been corrected as of 13 June 2016. The text is incorrect in the printed version of the journal. 